\title{
THE EXACT DEFINITION OF FUZZY RANDOMNESS: AN APPLICATION OF THE MATHEMATICS OF PARTIAL PRESENCE
}

\author{
Supahi Mahanta, Department of Statistics, Gauhati University, Guwahati-781014, Assam, India \\ supahi_mahanta@rediffmail.com
}

Rituparna Chutia, Department of Mathematics, Gauhati University, Guwahati-781014, Assam, India Rituparnachutia7@rediffmail.com

Hemanta K. Baruah, Department of Statistics, Gauhati University, Guwahati-781014, Assam, India hemanta_bh@yahoo.com

\begin{abstract}
Fuzzy randomness leads to fuzzy conclusions. Such fuzzy conclusions can indeed be made in terms of probability. In this article, the concept of fuzzy randomness has been discussed using the mathematics of partial presence. Two important points have been suggested in this article. First, fuzzy randomness should be explained with reference to the Randomness - Fuzziness Consistency Principle, and only then the mathematical explanations of fuzzy randomness would actually be complete. Secondly, in every case of fuzzy statistical hypothesis testing, the alternative hypotheses must necessarily be properly defined. The authors in this article have described fuzzy randomness with reference to a numerical example of using the Student's t-test statistic.
\end{abstract}

Keywords: Complement of a fuzzy set, the Randomness - Fuzziness Consistency Principle, Student's t-statistic.

\section{Introduction}

Fuzzy randomness arises when the random variables cannot be observed with exactness. Fuzzy randomness in terms of uncertain probabilities has been studied by Buckley and Eslami (2003, 2004) and Buckley (2003), among others. With reference to testing of statistical hypotheses, Goswami et al (1997) and Talukdar and Baruah (2007, 2010a, 2010b, 2010c, 2011) have studied randomness with fuzzy observations. Goswami and Baruah (2008a) studied the effect of fuzziness on the binomial probability law. Fuzzy time series analysis was studied by Goswami and Baruah (2007, 2008b). In all these cases, the parameters concerned were taken to be fuzzy numbers, and the statistical analytical matters were dealt with accordingly.
However, based on a set operation called superimposition (Baruah, 1999a), it has meanwhile been established by Baruah (2010a, 2010b, 2011b, 2011c, 2011d, 2012) that every law of fuzziness can actually be expressed in terms of two laws of randomness, with randomness defined in the measure theoretic sense, and accordingly fuzzy randomness should be explained with reference to two laws of randomness defined for every fuzzy observation. In this article, we are going to put forward the exact mathematical analysis of fuzzy randomness.

The Zadehian definition of complement of a fuzzy set is defective (Baruah, 1999b, 2011a). In the Zadehian definition of complementation, fuzzy membership function and fuzzy membership value have been taken to be the same, and that is where the defect lies. Indeed fuzzy membership function and fuzzy membership value are two different things for the complement of a normal fuzzy set (Baruah, 2011c). The membership function of the complement of a normal fuzzy number is 1 over the entire real line, with the condition that it is measured from the membership function of the normal fuzzy number concerned. In studying fuzzy randomness, while testing statistical hypotheses, the alternative hypotheses of fuzzy null hypotheses have always been wrongly defined. In this article, we would discuss regarding how an alternative hypothesis has to be stated. 
It should be noted that the notion of probability does not enter into the measure theoretic definition of a random variable (Rohatgi and Saleh, 2001, pages 41 - 43). When a variable is probabilistic, it has to be random by definition although when a variable is random, it need not be probabilistic. Accordingly, all results of the classical theory of probability are automatically applicable to a random variable defined in the measure theoretic sense. In the term 'fuzzy randomness', as available in the literature, the word 'randomness' has been taken as equivalent to the word 'probabilistic'.

In what, follows, we shall discuss in short Baruah's Randomness - Fuzziness Consistency Principle, and we shall state the axiom defining the complement of a fuzzy set. Thereafter we shall discuss how exactly to define fuzzy randomness. Finally, we shall discuss the matters with reference to testing a fuzzy hypothesis in the case of the Student's $t$-test.

\section{The Randomness - Fuzziness Consistency Principle}

A normal fuzzy number $N=[\alpha, \beta, \gamma]$ is an interval around the real number $\beta$ with the elements in the interval being partially present. Partial presence of an element in a fuzzy set is defined by the membership function. A normal fuzzy number $N=[\alpha, \beta, \gamma]$ is associated with a membership function $\mu_{N}$ $(x)$, where

$$
\mu_{N}(x)= \begin{cases}\psi_{1}(x), & \alpha \leq x \leq \beta \\ \psi_{2}(x), & \beta \leq x \leq \gamma \\ 0, & \text { otherwise }\end{cases}
$$

Here $\Psi_{1}(x)$ is continuous and nondecreasing in the interval $[\alpha, \beta]$, and $\Psi_{2}(x)$ is continuous and non-increasing in the interval $[\beta, \gamma]$, where

$$
\begin{aligned}
& \Psi_{1}(\alpha)=\Psi_{2}(\gamma)=0, \\
& \Psi_{1}(\beta)=\Psi_{2}(\beta)=1 .
\end{aligned}
$$

In the Dubois-Prade nomenclature, $\Psi_{1}(x)$ is called the Left Reference Function, and $\Psi_{2}(x)$ is called the Right Reference Function of the normal fuzzy number. Construction of a normal fuzzy number would depend on construction of these two reference functions. Defining the operation called Superimposition of Sets and using the Glivenko-Cantelli Theorem (Loeve, 1977) on Order Statistic, Baruah (2010a, 2010b, 2011b, 2011c, 2011d, 2012) has established the following result which we shall state as a theorem that uncovers the missing link between fuzziness and randomness, which was being searched for by the workers in fuzziness since 1965 . number

Theorem 1: For a normal fuzzy$$
N=[\alpha, \beta, \gamma]
$$

with membership function

$$
\mu_{N}(x)= \begin{cases}\psi_{1}(x), & \alpha \leq x \leq \beta \\ \psi_{2}(x), & \beta \leq x \leq \gamma \\ 0, & \text { otherwise }\end{cases}
$$

such that

$$
\begin{aligned}
& \Psi_{1}(\alpha)=\Psi_{2}(\gamma)=0, \\
& \Psi_{1}(\beta)=\Psi_{2}(\beta)=1,
\end{aligned}
$$

$\Psi_{1}(x)$ is the distribution function of a random variable defined in the interval $[\alpha, \beta]$, and $\Psi_{2}(x)$ is the complementary distribution function of another random variable defined in the interval $[\beta, \gamma]$.

It needs to be mentioned at this point that the Glivenko - Cantelli theorem on convergence of empirical probability distributions can actually be seen as the backbone of mathematical statistics. This theorem is about probability distribution functions, and therefore it will be applicable for distribution functions of random variables with randomness defined in the measure theoretic sense as well (Baruah, 2011b, 2012). As we have mentioned earlier, in the measure theoretic sense, if a variable is probabilistic, it has to be necessarily random, although when a variable is random, it does not have to be probabilistic.

It is known that a distribution function of a random variable is non-decreasing, and that a complementary distribution function of a random variable is non-increasing. The functions are continuous and differentiable. Differentiation of $\Psi_{1}(x)$ and $\left(1-\Psi_{2}(x)\right)$ would give two density functions. This means, one needs two laws of randomness, one in the interval $[\alpha, \beta]$ and the other in $[\beta, \gamma]$, to construct a normal fuzzy number $[\alpha, \beta, \gamma]$. 
For a triangular fuzzy number, differentiation of $\Psi_{1}(x)$ and $\left(1-\Psi_{2}(x)\right)$ would give two uniform density functions. It is well known that the uniform law of randomness is the simplest of all probability laws. Thus two uniform laws of randomness lead to the simplest fuzzy number. When we say that a normal fuzzy number is of the triangular type, it actually means that we have assumed that the left reference function is a uniform distribution function and the right reference function is a uniform complementary distribution function. We have actually found that the triangular fuzzy number appears very naturally in defining fuzziness (Das, 2013).

Theorem - 1 can actually be called the Randomness - Fuzziness Consistency Principle (Baruah, 2010a, 2010b, 2011c). Thus according to this principle, the DuboisPrade left reference function is actually a distribution function by definition and similarly the right reference function is nothing but a complementary distribution function. In other words, two laws of randomness, probabilistic or otherwise, are not only necessary but also sufficient to define a law of fuzziness.

\section{The Complement of a Fuzzy Set}

In the Zadehian definition of the complement of a fuzzy set, fuzzy membership function and fuzzy membership value are taken to be the same, which led to the conclusion that the fuzzy sets do not follow the set theoretic axioms of exclusion and contradiction. For the complement of a normal fuzzy set, fuzzy membership function and fuzzy membership value are two different things, and the complement of a normal fuzzy set has to be defined accordingly.

If a normal fuzzy number $N=[\alpha, \beta, \gamma]$ is defined with a membership function $\mu_{N}(x)$, where

and

$$
\begin{array}{r}
\mu_{N}(x)=\Psi_{1}(x), \text { if } \alpha \leq x \leq \beta \\
=\Psi_{2}(x), \text { if } \beta \leq x \leq \gamma
\end{array}
$$

where

$$
=0 \text {, otherwise, }
$$

$$
\begin{aligned}
& \Psi_{1}(\alpha)=\Psi_{2}(\gamma)=0, \\
& \Psi_{1}(\beta)=\Psi_{2}(\beta)=1,
\end{aligned}
$$

the complement $N^{C}$ will have the membership function $\mu_{N}^{C}(x)$, where

$$
\mu_{N}{ }^{C}(x)=1,-\infty<x<\infty,
$$

with the condition that $\mu_{N}^{C}(x)$ is to be counted from $\Psi_{1}(x)$, if $\alpha \leq x \leq \beta$, from $\Psi_{2}(x)$, if $\beta \leq x \leq \gamma$, and from 0 , otherwise, so that we keep a difference between the fuzzy membership function and the fuzzy membership value. Baruah (1999b, 2011c) has forwarded this definition of the complement of a fuzzy set which is based on the following axiom:

Axiom 1: The fuzzy membership function of the complement of a normal fuzzy number $N$ is equal to 1 for the entire real line, with the membership value counted from the membership function of $N$.

\section{The Exact Definition of Fuzzy Randomness}

In studying fuzzy randomness, the workers used the definition of complement of a fuzzy set to frame the alternative hypotheses with reference to the fuzzy null hypotheses. Whenever a fuzzy null hypothesis was found rejectable, this wrong definition came into picture.

While making the statistical conclusions with reference to fuzzy random data, there was another lack. If the two laws of randomness defining fuzziness are indeed laws of probability, two possibilities can actually be there. When a non-rejectable hypothesis is fuzzified, there would still be a probability that the fuzzy hypothesis would actually be found rejectable, the probability of rejection decided by the right reference function. In the same way, if a rejectable hypothesis is fuzzified, there would still be a probability that the fuzzy hypothesis would be found non-rejectable, the probability of nonrejection being decided by the left reference function this time (Baruah, 2011c).

Assume that $X$ is a random variable following the normal probability law with mean $\mu$ and variance unity. Now if the parameter $\mu$ is fuzzy, with membership defined in $[\mu-\delta, \mu, \mu+\delta]$, we would actually define an infinite number of normal probability density functions with location 
parameter ranging from $(\mu-\delta)$ to $(\mu+\delta)$ with maximum membership assigned at the value $\mu$. This is where the current definition of fuzzy randomness ends.

Assume now that we have a normally distributed population with mean $\mu$ and variance $\sigma^{2}$. From this population, a sample of $n$ observations $x_{1}, x_{2}, \ldots, x_{n}$ has been drawn, and we can then proceed to infer about the population, based on the sample data. Assume further that we have fuzzy data and we need to proceed for statistical analysis with reference to fuzzy randomness.

The data are in terms of fuzzy numbers around $x_{i}, i=1,2, \ldots, n$ defined as, say,

$$
X_{\mathrm{i}}=\left[x_{i}-\delta, x_{i}, x_{i}+\delta\right], \delta \geq 0 \text {. }
$$

The analysis can now proceed accordingly. Without loss of generality, and for computational simplicity, such fuzzy numbers are usually taken as triangular.

It can be seen that the equivalence of the definitions of the Dubois-Prade left reference function $\Psi_{1}(x), \alpha \leq x \leq \beta$, and a distribution function gives us

$$
\frac{d}{d x} \psi_{1}(x)=\varphi_{1}(x) \text {, say }
$$

where

$$
\int_{\alpha}^{\beta} \varphi_{1}(x) d x=1
$$

In the same way, the equivalence of the definitions of the Dubois-Prade right reference function $\Psi_{2}(x), \beta \leq x \leq \gamma$, and a complementary distribution function, gives us

where

$$
\frac{d}{d x}\left(1-\psi_{2}(x)\right)=\varphi_{2}(x), \text { say, }
$$

$$
\int_{\beta}^{\gamma} \varphi_{2}(x) d x=1
$$

Now, according to Baruah's Randomness - Fuzziness Consistency Principle, a triangular fuzzy number of the type

$$
X_{i}=\left[x_{i}-\delta, x_{i}, x_{i}+\delta\right]
$$

with membership function

$$
\mu_{X_{i}}(x)=\left\{\begin{array}{l}
\frac{x-x_{i}+\delta}{\delta}, \text { if } x_{i}-\delta \leq x \leq x_{i}, \\
\frac{x_{i}+\delta-x}{\delta}, \text { if } x_{i} \leq x \leq x_{i}+\delta, \\
0, \text { otherwise }
\end{array}\right.
$$

is in fact defined by two laws of randomness with distribution functions

$$
F_{1}(x)=\frac{x-x_{i}+\delta}{\delta} \text {, if } x_{i}-\delta \leq x \leq x_{i}
$$

and

$$
F_{2}(x)=1-\frac{x_{i}+\delta-x}{\delta} \text {, if } x_{i} \leq x \leq x_{i}+\delta,
$$

so that their densities

$$
\frac{d}{d x} F_{1}(x)=\frac{1}{\delta}, \text { if } x_{i}-\delta \leq x \leq x_{i},
$$

and

$$
\frac{d}{d x} F_{2}(x)=\frac{1}{\delta}, \text { if } x_{i} \leq x \leq x_{i}+\delta
$$

are uniform.

Accordingly, fuzzy randomness should be defined as follows. First, there should be a variable following some law of probability. Secondly, around every realization of the probabilistic variable, there should be fuzziness. This fuzziness in turn will be explained by two laws of randomness, with randomness defined in the measure theoretic sense. If it is presumed that the two laws of randomness are in fact two laws of probability, then the conclusions can be made probabilistically.

\section{Student's $-t$ Test with Fuzzy Data}

Assume that a random sample of five students has been collected from a normal population and their heights are measured. The heights of the five students are 63, 67, 70, 71 and 73 inches. Let us assume that mean height of the population is 66 inches.

Now, we want to test whether the data are consistent with the assumption of a mean height of 66 inches in the population, i.e., $\mathrm{H}_{0}$ : $\mu=66$, against the alternative hypothesis, $H_{1}$ : $\mu \neq 66$.

Under $\mathrm{H}_{0}$, the test statistic is given by

$$
t=\frac{\bar{x}-\mu_{0}}{s^{2} / \sqrt{n-1}},
$$

which follows the Student's $-t$ probability distribution with (n-1) degrees of freedom, where $\bar{x}$ is the sample mean and $s^{2}$ is the sample variance.

Here, the calculated value of $t$ is 1.4364 which is less than the tabulated value of $t$ i.e. 2.78 at $5 \%$ probability level of 
significance for 4 degrees of freedom. Therefore, we may conclude that there is no reason to reject the null hypothesis that the sample has come from a population with mean height 66 inches.

In a fuzzy situation, let us start with data of the type $\left[x_{i}-1, x_{i}, x_{i}+1\right]$ with an assumption that the data are triangular. The random variable $X$ of which $x$ is a realization in the sample was assumed to be normally distributed. In other words, we would start with an assumption that the two laws of randomness, one on $\left[x_{i}-1, x_{i}\right]$ and the other on $\left[x_{i}, x_{i}+1\right]$, are uniform, for a normally distributed realization $x$ with mean $\mu$ and error variance $\sigma^{2}$, say. We would presume further that these two laws of randomness are indeed two laws of probability so that we can infer probabilistically.

Thus, we have the heights of the students with triangular membership functions as [62, 63, 64], [66, 67, 68], [69, 70, 71], [70, 71, 72], [72, 73, 74]. The null hypothesis would be

$\mathrm{H}_{0}$ : the data of interval type are consistent with the assumption of a fuzzy mean height of $[65,66,67]$ inches in the population, i.e.,

$$
\mathrm{H}_{0}: \mu=[65,66,67] \text {. }
$$

The alternative hypothesis is,

$$
\mathrm{H}_{1}: \mu=[65,66,67]^{\mathrm{C}} \text {, }
$$

where the complement should be defined as discussed earlier.

Under $\mathrm{H}_{0}$, we have obtained the fuzzy value of Student's - $t$ with the following fuzzy membership function

$$
\mu_{t}(x)=\left\{\begin{array}{cc}
\frac{\sqrt{\left(12.8+20.2 x^{2}\right)^{2}-64\left(2.56-35.4 x^{2}\right)}-\left(12.8+20.2 x^{2}\right)}{32} & 0.2689 \leq x \leq 1.4364 \\
\frac{\left(76.8+11.2 x^{2}\right)-\sqrt{\left(76.8+11.2 x^{2}\right)^{2}-64\left(92.16-4 x^{2}\right)}}{32} & 1.4364 \leq x \leq 4.8 \\
0, & \text { otherwise }
\end{array}\right.
$$

Now, this fuzzy number gives the following two distribution functions according to the Randomness-Fuzziness Consistency Principle:

$$
\psi_{1}(x)=\frac{\sqrt{\left(12.8+20.2 x^{2}\right)^{2}-64\left(2.56-35.4 x^{2}\right)}-\left(12.8+20.2 x^{2}\right)}{32},
$$

and

$$
\left(1-\psi_{2}(x)\right)=1-\frac{\left(76.8+11.2 x^{2}\right)-\sqrt{\left(76.8+11.2 x^{2}\right)^{2}-64\left(92.16-4 x^{2}\right)}}{32 \quad 1.4364 \leq x \leq 4.8},
$$

This means, the fuzzy value of

$$
t=[0.2689,1.4364,4.8]
$$

with left and right reference functions $\psi_{1}(x)$ and $\psi_{2}(x)$ defined in $0.2689 \leq \mathrm{x} \leq$ 1.4364 and $1.4364 \leq \mathrm{x} \leq 4.8$ respectively, would be defined by the two densities $\frac{d}{d x} \psi_{1}(x)$ and $\frac{d}{d x}\left(1-\psi_{2}(x)\right)$ in the respective ranges.

We now proceed to look into the matters of making a fuzzy conclusion statistically. The tabulated non-fuzzy value of $t$ at 5\% level of significance for 4 degrees of freedom is 2.78 , which lies between 1.4364 to 4.8 .

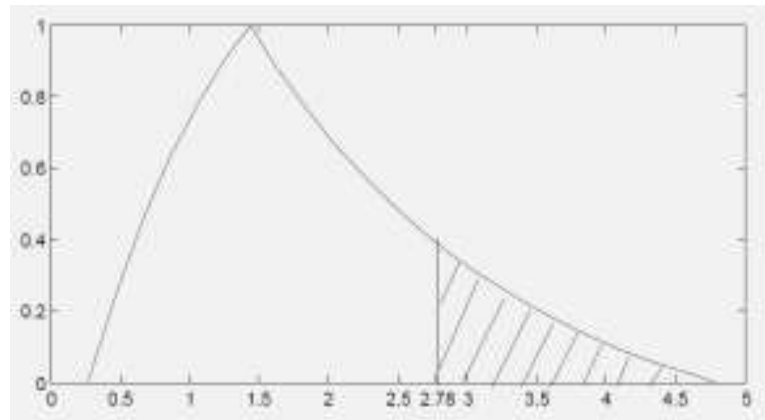

Figure: The Membership Curve

To the right of the tabulated value of $t$, i.e., 2.78, the area under the probability density function of Student's $t$ is 0.025. 2.78 is on that part of the interval on which the right reference function is defined. Now, in our perspective the probability density function concerned with the right reference function is given by

$$
\begin{array}{r}
\frac{d}{d x}\left(1-\psi_{2}(x)\right)=-\frac{22.4 x}{32}+\frac{22.4 x\left(76.8+11.2 x^{2}\right)+256 x}{32 \sqrt{\left(76.8+11.2 x^{2}\right)^{2}-64\left(92.16-4 x^{2}\right)}}, \\
1.4364 \leq x \leq 4.8
\end{array}
$$

Therefore, the probability that $t \geq 2.78$ would be the area under this probability density function for $t \geq 2.78$, which is the area of the right tail beyond 2.78. The area of the left tail from 1.4364 to 2.78 is $\left(1-\psi_{2}(2.78)\right)$. Thus the area of the right tail is $\psi_{2}(2.78)$ again, which is nothing but the membership value of $t$ at 2.78. $\psi_{2}(2.78)=0.3960$ is 
therefore the probability that the fuzzy null hypothesis that the sample has come from a population with fuzzy mean height 66 inches would have to be rejected at $5 \%$ probability level of significance. In other words, when a non-rejectable hypothesis is fuzzified, there will still be a probability that the fuzzy hypothesis would actually be found rejectable. In the same way, if a rejectable hypothesis is fuzzified, there would still be a probability that the fuzzy hypothesis would be found non-rejectable, the probability of nonrejection being decided by the left reference function this time.

\section{Conclusions}

Partial presence of an element in a normal fuzzy number can be expressed in terms of two laws of randomness. The membership function of a normal fuzzy number $[\alpha, \beta, \gamma]$ is actually a distribution function in $[\alpha, \beta]$ and a complementary distribution function in $[\beta, \gamma]$. Based on this principle, the exact definition of fuzzy randomness has been forwarded in this article. Further, the complement of a fuzzy set should be redefined. In testing of fuzzy hypothesis, we deal with the alternative hypothesis which is the complement of the fuzzy null hypothesis. One should note that for the complement of a normal fuzzy set, fuzzy membership function and fuzzy membership value are two different things. We have shown that when a non-rejectable hypothesis is fuzzified, there would still be a probability that the fuzzy hypothesis would actually be found rejectable. In the same way, if a rejectable hypothesis is fuzzified, there would still be a probability that the fuzzy hypothesis would be found non-rejectable.

\section{Reference}

1. Baruah, H. K. (1999a), Set Superimposition and Its Application to the Theory of Fuzzy Sets, Journal of the Assam Science Society, Vol. 40, Nos. 1 \& 2, $25-31$.

2. Baruah, H. K. (1999b), Fuzzy Membership with respect to a Reference Function, Journal of the Assam Science Society, Vol. 40, No. 3, $65-73$.
3. Baruah, H. K. (2010a), The randomness fuzziness consistency principle, International Journal of Energy, Information and Communications, Vol. 1, Issue 1, $37-48$.

4. Baruah, H. K. (2010b), The RandomnessFuzziness Consistency Principle, Invited Article, International Congress of Mathematics, Proceedings of the Satellite International Conference on Probability and Statistics, Sambalpur University, India.

5. Baruah, H. K. (2011a), Towards Forming a Field of Fuzzy Sets, International Journal of Energy, Information and Communications, Vol. 2, Issue 1, $16-$ 20.

6. Baruah, H. K. (2011b), Construction of the membership function of a fuzzy number, ICIC Express Letters, Vol. 5, Issue 2, 545-549.

7. Baruah, H. K. (2011c), The theory of fuzzy sets: beliefs and realities, International Journal of Energy Information and Communications, Vol. 2, No. 2, 1-22.

8. Baruah, H. K. (2011d), In search of the root of fuzziness: the measure theoretic meaning of partial presence, Annals of Fuzzy Mathematics and Informatics, Vol. 2, No. 1, 57-68.

9. Baruah, H. K. (2012), Construction of normal fuzzy numbers using the mathematics of partial presence, Journal of Modern Mathematics Frontier, Vol. 1, No. 1, 9-15.

10. Buckley, J. J. (2003), Uncertain probabilities III: the continuous case, Soft Computing - A Fusion of Foundations, Methodologies and Applications, Vol. 8, No. 3, 200 - 206.

11. Buckley, J. J. and Eslami, E. (2003), Uncertain probabilities I: the discrete case, Soft Computing - A Fusion of Foundations, Methodologies and Applications, Vo. 7, No. 8, $500-505$.

12. Buckley, J. J. and Eslami, E. (2004), Uncertain probabilities II: the continuous case, Soft Computing - A Fusion of Foundations, Methodologies and Applications, Vol. 8, No. 3, 193 - 199.

13. Das, D., Mahanta S., Chutia, R. and Baruah, H. K. (2013), Construction of normal fuzzy numbers: case studies with Indian stock exchange data, Annals of Fuzzy Mathematics and Informatics (in press).

14. Goswami, P. and Baruah, H. K. (2008a), Fuzzy Discrete Distributions: the Binomial Case, Journal of Fuzzy Mathematics, Vol. 16, No. 3, 671 676.

15. Goswami, P. and Baruah, H. K. (2008b), The Fuzzy ARIMA $(1,1)$ Process, Vol. 16, No. 3, 721 729.

16. Goswami, P. and Baruah, H. K., (2007), Fuzzy Time Series Analysis, Journal of Fuzzy Mathematics, Vol. 15, No. 3, 513 -523.

17. Goswami, P., Dutta, P. and Baruah, H. K. (1997), The Latin Square Design Using Fuzzy Data, Journal of Fuzzy Mathematics, Vol. 5, No. 4, 767 -779.

18. Loeve, M. (1977), Probability Theory, Springer Verlag, New York.

19. Rohatgi V. K. and Saleh A. K. E. (2001), An Introduction to Probability and Statistics, Second 
Edition, Wiley Series in Probability and Statistics, John Wiley \& Sons (Asia) Pte Ltd., Singapore.

20. Talukdar, R. and Baruah, H. K. (2007), Sequential Probability Ratio Test with Fuzzy Observations, Journal of Fuzzy Mathematics, Vol. 15, No. 3, $627-643$.

21. Talukdar, R. and Baruah, H. K. (2010a), A Fuzzy Sequential Decision Procedure Applied to Completely Randomized Design of Experiments, International Journal of Contemporary Mathematical Sciences, Vol. 5, No. 26, 1283 - 1302.
22. Talukdar, R. and Baruah, H. K. (2010b), A Sequential Variance Ratio Test with Fuzzy Observations, Advances in Fuzzy Mathematics, Vol. 5, No. 3, 301-309.

23. Talukdar, R. and Baruah, H. K. (2010c), A Two Sample Sequential t-Test with Fuzzy Observations, Applied Mathematical Sciences, Vol. 4, No. 68, 3361 - 3374.

24. Talukdar, R. and Baruah, H. K. (2011), A Sequential Multi-Decision Procedure with Fuzzy Observations, Journal of Fuzzy Mathematics, Vol. 19, No. $1,125-140$. 